\title{
Sharon Shiovitz-Ezra
}

\section{Confidant networks and loneliness}

- Loneliness exists in all of the SHARE Wave 4 countries

- Older people who have no confidants are also the loneliest

- Having a spouse as a confidant is the most influential factor against loneliness

\subsection{The phenomenon of loneliness}

Reports on feelings of loneliness have been found across different countries and societies. In that sense, loneliness can be considered a universal phenomenon (Perlman 2004). However, the prevalence of loneliness varies across countries, and even in countries on the same continent. For instance, a contemporary study that addressed the prevalence of loneliness across Europe using SHARE Wave 1 data revealed that the prevalence of loneliness among older adults aged 65 and over ranged from only 25 percent in Denmark to more than 60 percent in Greece (Sundström et al. 2009). A similar trend was found among people aged 50 and over in a more recent study that used data from SHARE Wave 2 (2006-2007) (Fokkema et al. 2012). The findings from both studies revealed that loneliness is more prevalent in Southern countries, which have a familistic orientation, than in Northern countries, where people are more individualistic. This counter-intuitive trend is consistent with the results of other studies, and is often attributed to the different social expectations that prevail in different social contexts. That is, in more familistic, collectivist societies there are higher expectations of connectedness that can often lead to feelings of loneliness, in light of the social losses experienced more frequently in old age (Johnson \& Mullins 1987).

The growing interest in loneliness among researchers, practitioners, and policy makers derives not only from its prevalence but also from consistent evidence of the negative consequences that accompany loneliness. There is an abundance of findings attesting to the deleterious emotional and physical consequences of loneliness, which include depressive symptomatology (Van der Weele et al. 2011), sedentary life style in old age (Netz et al. 2012) and increased odds for all-cause mortality (Shiovitz-Ezra \& Ayalon 2010).

Loneliness is defined as a sense of distress caused by perceived dissatisfaction with one's social relationships. The sense of subjective dissatisfaction derives from a gap between desired social relationships and the relationships 
that one actually has (Peplau \& Perlman 1982). According to the deficit model (Perlman \& Peplau 1998), feelings of loneliness are experienced when one's social needs are not met. The perceived social deficits can be in terms of quantity (e. g., a limited number of social members and infrequent social contacts) and especially in terms of quality. It has been argued that qualitative assessments of social relationships are more closely linked to well-being outcomes such as loneliness than are objective aspects such as the nature of one's social environment (Cutrona 1982). De Jong Gierveld (1987) proposed a theoretical model of loneliness which underscores the importance of the qualitative aspect of social networks. The model encompasses two sets of characteristics as potential determinants of loneliness. The first includes objective characteristics of the social network, such as its size; and the second relates to subjective evaluations of the social network, which include the perceived availability of support and satisfaction with social contacts.

Several empirical studies, as well as Pinquart and Sorensen's meta-analysis (2001), have supported the argument that subjective assessments play a more important role in determining loneliness than do objective characteristics. In one study, which was conducted among a representative sample of older Americans aged 57-85, the quality of social relationships was measured on the basis of perceived support and strain from family members and friends. These variables explained a significantly higher amount of the variance in loneliness of married and unmarried respondents than did the objective social network indicators (Shiovitz-Ezra \& Leitsch 2010). Moreover, married persons were protected against loneliness only when the spouse was defined as a confidant (Hawkley et al. 2008). Similarly, both negative and positive markers of marital quality were found to be closely associated with loneliness (de Jong Gierveld et al. 2009).

However, the above-mentioned studies showed that the importance of subjective assessments of social relationships is limited by the number and variety of subjective indicators that exist. Most studies have employed between 2 to 6 indicators relating to limited aspects of social network quality. SHARE Wave 4 used the name generator method, which enables in-depth empirical examination of confidant network characteristics and their associations with loneliness. As in the National Social Life, Health and Aging Project (NSHAP), SHARE Wave 4 participants were asked to name people with whom they discussed important matters. This screening question was followed by a series of questions that included detailed information on the confidant network members listed in the name generator. These included questions about the participant's relationship with the confidant member (e.g., spouse, child, grandchild, friend, etc.); how close the participant feels to the confidant; and how close the confidant lives to the participant. Therefore, the name generator produced a broad spectrum of qualitative measures. 
The current study was based on the new SHARE 4 data. Based upon previous findings, it was hypothesised that older adults who reported having no confidant network would experience greater loneliness. Furthermore, the study evaluated the relative importance of different qualitative measures among older adults with confidant networks.

\subsection{Studying loneliness}

The study employed data collected in SHARE Wave 4 (2010) among older people aged 50 and over in 15 countries $(N=50,552)$. This allowed us to assess loneliness in a large number of European countries, and provided a basis for expanding current knowledge about the phenomenon. Although Estonia participated in the SHARE Wave 4 countries, its drop-off data were not available in Release 1. Insofar as the loneliness measures were asked in the drop-off questionnaire, Estonian respondents were not included as part of the present analysis.

\subsection{The measures employed in this study}

Loneliness. Loneliness was measured through the short form of the well-known R-UCLA loneliness scale. Participants were asked how much of the time they felt a sense of being left out, a lack of companionship, and isolation on a 3-point scale ranging from 1 (hardly ever) to 3 (often). The three items were summed up to create a scale ranging from 3-9. Higher scores represented a greater extent of loneliness. In the SHARE Wave 4 sample, the internal reliability of the loneliness scale was found to be sufficient $(\alpha=.78)$.

Confidant network indicators. In the current study, several variables were derived to measure confidant networks: confidant network size (based on counting the names of the social network members listed in the roster); the composition of the social network (using six categorical variables to represent different network members: spouse, children, grandchildren, parents, siblings, and friends); global satisfaction with the social network; average proximity and frequency of contact with social network members, and average emotional closeness with social network members.

Demographic variables. Age (>50), gender (men/women), and country were control variables. 


\subsection{The means of analysis in the present study}

The loneliness mean was calculated across SHARE countries. ANOVAs were used to examine country differences. Among participants who reported having a confidant network, bivariate associations between network measures and loneliness were analysed using unadjusted linear regressions. In the adjusted model, all confidant network measures were included, with age, gender, and country as controls. In the unadjusted and adjusted models, the reference categories for the social network composition measures were the participants without a given type of relationship. For example, "no children" served as the reference category for "children" in the confidant social network. In the case of parents and friends in the network, the reference categories were no confidant parents and friends, because the question of "having parents who are alive" was not asked of all respondents and "having friends" was not asked at all. The statistical program STATA 10 was used for the analysis.

\subsection{How prevalent is loneliness in Europe and what explains it?}

Table 30.1 presents the mean for loneliness among the overall SHARE Wave 4 sample of persons aged 50+, and across the 15 European countries that participated in SHARE Wave 4 and had loneliness data. The overall mean for loneliness was relatively low $(M=3.73$, St. $D=1.26)$, although the loneliness scores varied across countries. The three nations at the top of the table - the Czech Republic, Hungary, and Italy - showed the highest mean scores for loneliness. Conversely, the lowest mean scores for loneliness were found in Switzerland, Austria, and Denmark. Scheffe post-hoc tests revealed that the differences between the top and bottom countries were significant.

Bivariate associations between confidant network measures and loneliness are presented in Table 30.2. First, the association of having a confidant network (as opposed to not having a confidant network) with feelings of loneliness was examined (not shown). People aged 50 and over without a confidant network were significantly more lonely $(M=4.01 ; S E=.04,95 \% C I=3.97,4.13)$ than their counterparts with a confidant network of any size $(M=3.42 ; S E=.00,95 \% C I$ $=3.41,3.43$ ). In the next stage, associations between confidant network characteristics and loneliness were examined among people who reported having one or more confidants. Regarding the composition of the confidant network, both positive and negative associations with loneliness were found. Compared to 
Table 30.1: Mean of loneliness across European countries

\begin{tabular}{llll}
\hline Country & N & Mean & St.D \\
\hline 1. CZ & 4,126 & 4.11 & 1.29 \\
2. HU & 2,759 & 4.08 & 1.54 \\
3. IT & 3,123 & 4.07 & 1.55 \\
4. BE & 3,689 & 3.97 & 1.39 \\
5. PL & 1,441 & 3.85 & 1.40 \\
6. FR & 4,609 & 3.84 & 1.39 \\
7. SE & 1,652 & 3.67 & 1.09 \\
8. PT & 1,292 & 3.66 & 1.31 \\
9. SI & 2,570 & 3.59 & 1.06 \\
10. ES & 3,263 & 3.54 & 1.18 \\
11. DE & 1,329 & 3.52 & 1.00 \\
12. NL & 2,402 & 3.50 & .99 \\
13. DK & 1,874 & 3.45 & .95 \\
14. AT & 4,343 & 3.45 & .98 \\
15. CH & 3,533 & 3.38 & .91 \\
Overall 50+sample & 42,005 & 3.73 & 1.26 \\
\hline
\end{tabular}

Notes: Excluding respondents aged $<50$ and respondents with no loneliness data. The loneliness scale range is $3-9$, higher score presents greater loneliness.

Source: SHARE Wave 4 release 1

people with no children, having a confidant child was negatively associated with loneliness. Nonetheless, having no children in the confidant network was still negatively associated with loneliness. This trend repeated itself with regard to siblings and spouses. However, the strongest negative association with loneliness ( $\beta=-.65, p<.001)$ was found among those with a confidant spouse. In other words, people with a confidant spouse felt least lonely. Similarly, people with a confidant friend, were less lonely than people with no confidant friends. Surprisingly, people without grandchildren felt less lonely than people with grandchildren regardless of whether or not the grandchild was mentioned in the confidant network.

Other confidant network characteristics were found to be variously associated with loneliness. Network size, emotional closeness and satisfaction with the network were negatively associated with loneliness. That is, the greater the number of confidants, the closer they were emotionally and the greater the satisfaction with them, the less the sense of loneliness was. However, more frequent contact and greater physical proximity to one's confidants were both positively associated with loneliness. 
Table 30.2: Confidant social network characteristics: Descriptive and bivariate analysis (sample size varies across variables)

\begin{tabular}{|c|c|c|c|}
\hline & $\begin{array}{l}\text { Frequencies } \\
\%\end{array}$ & $\begin{array}{l}\text { Mean } \\
\text { (SE), [95\%Cl] }\end{array}$ & $\begin{array}{l}\text { Loneliness } \\
\beta \text { (SE) }\end{array}$ \\
\hline No children - ref. ${ }^{1}$ & 9.3 & & \\
\hline Confidant child & 52.7 & & $-.21(.02)^{\star \star \star}$ \\
\hline No confidant child & 38.0 & & $-.34(.02)^{\star \star \star}$ \\
\hline No spouse - ref. & 26.5 & & \\
\hline Confidant spouse & 61.2 & & $-.65(.01)^{\star \star \star}$ \\
\hline No confidant spouse & 12.3 & & $-.38(.02)^{\star \star \star}$ \\
\hline No grandchildren - ref. & 31.8 & & \\
\hline Confidant grandchild & 3.7 & & $.23(.03)^{\star \star \star}$ \\
\hline No confidant grandchild & 64.5 & & $.03(.01)^{\star \star}$ \\
\hline No sibling -ref. & 19.0 & & \\
\hline Confidant sibling & 17.8 & & $-.11(.02)^{\star \star \star}$ \\
\hline No confidant sibling & 63.2 & & $-.15(.02)^{\star \star \star}$ \\
\hline No confidant parent - ref. & 93.9 & & \\
\hline Confidant parent & 6.0 & & $-.05(.03)$ \\
\hline No confidant friend - ref. & 69.8 & & \\
\hline Confidant friend & 30.2 & & $-.02(.01)$ \\
\hline Size of the $\mathrm{SN}^{2}$ & & $2.48(.01),[2.46,2.49]$ & $-.06(.00)^{\star \star \star}$ \\
\hline Proximity with SN & & 3.14(.01), [3.13,3.16] & $.07(.00)^{\star \star \star}$ \\
\hline Freq. of contact with SN & & 1.86(.00), $[1.85,1.87]$ & $.10(.01)^{\star \star \star}$ \\
\hline Satisfaction with SN & & $8.86(.01),[8.85,8.88]$ & $-.15(.00)^{\star \star \star}$ \\
\hline Emotional closeness with SN & & $3.30(.00),[3.29,3.31]$ & $-.20(.01)^{\star \star \star}$ \\
\hline
\end{tabular}

Significance: ${ }^{\star \star *}=1 \%$; ${ }^{* *}=5 \%$

Notes: Excluding respondents aged $<50$ and without a confidant social network. ${ }^{1}$ ref. - is abbreviation for reference group; ${ }^{2} \mathrm{SN}$ - is abbreviation for social network.

Source: SHARE Wave 4 release 1

In the adjusted model, all confidant network characteristics were taken into account as well as the control variables (age, gender, and country). The dominance of having a spouse (confidant or not) in the relationship with loneliness was still evident after the control variables and other network characteristics were taken into account. Having a spouse was significantly negatively associated with loneliness compared to not having a spouse. Moreover, a confidant spouse was found to be the most protective factor against loneliness in the model. Other characteristics that were negatively associated with loneliness were having children, and having a confidant friend. With regard to children, having children who were not mentioned in the confidant network was more protective against loneliness than having a child/children in the confidant 
network. Moreover, having grandchildren in the confidant network was associated with greater loneliness.

Other characteristics of the confidant network were also found to be either negatively or positively associated with loneliness. Whereas a larger confidant network, higher satisfaction, and greater emotional closeness with the social network decreased feelings of loneliness, more frequent contact with confidant members and greater proximity to them increased loneliness.

Table 30.3: Confidant social network correlates of loneliness: Multivariate analysis $(\mathrm{N}=39,186)$

\begin{tabular}{lll}
\hline & Beta coefficient (SE) & $95 \% \mathrm{Cl}$ \\
\hline No children - ref. ${ }^{1}$ & & \\
Confidant child & $-.06(.02)^{\star \star \star}$ & {$[-.11,-.07]$} \\
No confidant child & $-.13(.02)^{\star \star \star}$ & {$[-.18,-.08]$} \\
No spouse- ref. & & \\
Confidant spouse & $-.48(.02)^{\star \star \star}$ & {$[-.51,-.45]$} \\
No confidant spouse & $-.37(.02)^{\star \star \star}$ & {$[-42,-.33]$} \\
No grandchildren - ref. & & \\
Confidant grandchild & $.08(.03)^{\star \star}$ & {$[.01, .15]$} \\
No confidant grandchild & $.01(.02)$ & {$[-.02, .04]$} \\
No sibling - ref. & & \\
Confidant sibling & $-.02(.02)$ & {$[-.06, .02]$} \\
No confidant sibling & $-.00(.02)$ & {$[-.03, .03]$} \\
No confidant parent - ref. & & \\
Confidant parent & $.03(.03)$ & {$[-.02, .08]$} \\
No confidant friend - ref. & & \\
Confidant friend & $-.08(.02)^{\star \star \star}$ & {$[-.11,-.05]$} \\
Size of the SN ${ }^{\star *}$ & $-.04(.01)^{\star \star \star}$ & {$[-.06,-.03]$} \\
Proximity with SN & $.01(.01)^{\star \star}$ & {$[.00, .02]$} \\
Freq. of contact with SN & $.05(.01)^{\star \star \star}$ & {$[.03, .07]$} \\
Satisfaction with SN & $-.13(.01)^{\star \star \star}$ & {$[-.14,-.13]$} \\
Emotional closeness with SN & $-.06(.01)^{\star \star \star}$ & {$[-.08,-.04]$} \\
$R^{2}$ & 0.12 & \\
\hline & & \\
\hline
\end{tabular}

Significance: ${ }^{* *}=1 \%$; ${ }^{* *}=5 \%$

Notes: Controlled for age, gender and country. ${ }^{1}$ ref. - is abbreviation for reference group; ${ }^{2} \mathrm{SN}$ - is abbreviation for social network.

Source: SHARE Wave 4 release 1 


\subsection{Summary and conclusions}

The population examined in this chapter included 15 of 16 European countries from the SHARE Wave 4 sample. Three of the included countries were new participants in the SHARE survey, which thus provided a basis for expanding the examination of loneliness in Europe. Consistent with previous studies that addressed the prevalence of loneliness in different geographic locations (Perlman 2004), the data showed evidence of its occurrence in each of the 15 participating countries. On the whole, loneliness was more widespread in Southern and Eastern Europe than in Northern and Western European countries. This finding is consistent with a previous contemporary analysis of SHARE Wave 2 data (Fokkema et al. 2012), despite the different measures of loneliness used in Waves 2 and 4. Whereas loneliness was measured using the single direct measure adopted from the CES-D depression scale in SHARE Wave 2, the Wave 4 questionnaire introduced a more comprehensive scale of loneliness.

In addition, the name generator mechanism that was introduced in the fourth wave of SHARE provides more insights into loneliness, particularly regarding qualitative protective factors. Notably, people with no confidant network at all felt lonelier than people who had at least one confidant. Moreover, examination of different qualitative components of the confidant network revealed different patterns of effects on loneliness. The composition measures - particularly having a spouse - were most strongly associated with loneliness. The role of having a spouse as a dominant protective factor against loneliness has also been found in previous studies (e.g. Shiovitz-Ezra \& Leitsch 2010). However, having a confidant spouse with whom one can discuss important matters was the most influential factor. Having a confidant spouse was also negatively associated with loneliness in a recent study conducted in the U.S. (Hawkley et al. 2008).

Relationships with children and grandchildren, however, appear to be more complicated. Having a child (whether confidant or not) was negatively associated with loneliness compared to the "not having a child" status. However, not having a confidant child was a stronger negative predictor of loneliness. In other words, having a child who wasn't mentioned in one's confidant network was associated with feeling less lonely than having a confidant child. One possible explanation for this surprising finding is that although having children might serve as a protective factor against loneliness, when one's children are included in the confidant network there might be additional tension and conflicts in this close emotional relationship which, in turn, can reduce the protective impact of having a child on loneliness. In the same vein, the analysis revealed that having a confidant grandchild was positively associated with loneliness. It is possible that the need to consider one's grandchildren as con- 
fidants is indicative of restricted emotional relationships, which can increase feelings of loneliness.

Other confidant network indicators were also related to loneliness. Specifically, a larger confidant network, satisfaction with the network, and greater emotional closeness with the network were all related to lower feelings of loneliness. Negative associations between satisfaction with the social network and loneliness have also been found in a previous study (Hawkley et al. 2008). In contrast, frequent contact with confidants and living near them was associated with increased loneliness. These unexpected findings could be explained by the disadvantages of social relationships. Aside from the support that can be generated by close emotional contact, these relationships might also be accompanied by demands and criticism (Ruehlman \& Wolchik 1988). When the relationship with one's confidants is characterized by close physical proximity and frequent contact, the increased intensity of the relationships might lead to more negative emotions, which are frequently followed by greater loneliness. Ill health might also force older people to have emotionally close ties with those who live close by, but they are not necessarily pleased with this restriction.

The current study is restricted, to some degree, by its cross-sectional design, based only on SHARE Wave 4 data. It might be that lonely people establish different confidant networks. Therefore it will be useful to obtain longitudinal data in this area. When the next round of SHARE network data collection is completed, it may be possible to examine causal relationships between confidant networks and loneliness, yielding new insights into the phenomenon.

\section{References}

Cutrona, Carolyn (1982): "Transition to college: loneliness and the process of social adjustment”. In: Peplau, Letitia, Perlman, Daniel (Eds.): Loneliness: a sourcebook of current theory, research and therapy. New York: A Wiley-Interscience Publication, p. 291-309.

De Jong Gierveld, Jenny (1987): “Developing and testing a model of loneliness”. In: Journal of Personality and Social Psychology 53(1), p. 119-128.

De Jong Gierveld, Jenny, van Groenou, Marjolein, Hoogendoorn, Adrian, Smith, Johannes (2009): "Quality of marriages in later life and emotional and social loneliness". In: Journal of Gerontology: Social Sciences 64B(4), p. 497-506.

Fokkema, Tineke, De Jong Gierveld, Jenny, Dykstra, Pearl (2012): “Cross-national differences in older adult loneliness". In: The Journal of Psychology: Interdisciplinary and Applied 146(1-2), p. 201-228.

Hawkley, Louise, Hughes, Mary Elizabeth, Waite, Linda, Masi, Christopher, Thisted, Ronald, Cacioppo, John (2008): "From social structural factors to perceptions of relationship quality and loneliness: The Chicago Health, Aging, and Social Relations Study”. In: Journal of Gerontology: Social Sciences 63B(6), p. S375-S384. 
Johenson, Paul, Mullins, Larry (1987): “Growing old and lonely in different societies: toward a comparative perspective”. In: Journal of Cross Cultural Gerontology 2, p. 257-275.

Netz, Yael, Goldsmith, Rebecca, Shimony, Tal, Arnon, Michal, Zeev, Aviav (2012): “Loneliness is associated with an increased risk of sedentary life in older Israelis”. In: Aging and Mental Health, DOI: 10.108/13607863.2012.715140.

Peplau, Letitia, Perlman, Daniel (Eds.) (1982): Loneliness: a source book of current theory, research and therapy. New York: John Wiley.

Perlman, Daniel (2004): “European and Canadian studies of loneliness among seniors". In: Canadian Journal of Aging 23, p. 181-188.

Perlman, Daniel, Peplau, Letitia (1998): “Loneliness”. In: Friedman, Howart (Ed.): Encyclopedia of mental health. Vol. 2. San Diego, CA: Academic Press, p. 571-581.

Pinquart, Martin, Sörensen, Silvia (2001): “Influences on loneliness in older adults: a meta-analysis". In: Basic and Applied Social Psychology 23(4), p. 245-266.

Ruehlman, Linda, Wolchik, Sharlene (1988): “Personal goals and interpersonal support and hindrance as factors in psychological distress and well-being”. In: Journal of Personality and Social Psychology 55, p. 293-301.

Shiovitz-Ezra, Sharon, Ayalon, Liat (2010): "Situational versus chronic loneliness as risk factors for all cause mortality". In: International Psychogeriatrics 22, p. 455-462.

Shiovitz-Ezra, Sharon, Leitsch, Sara (2010): “The role of social relationships in predicting loneliness: The National Social Life, Health and Aging Project (NSHAP)". In: Social Work Research 34(3), p. 157-167.

Sundström, Gerdt, Fransson, Eleonor, Malmberg, Bo, Davey, Adam (2009): “Loneliness among older Europeans". In: European Journal of Ageing 6, p. 267-275.

Van der Weele, Tyler, Hawkley, Louise, Thisted, Ronald, Cacioppo, John (2011): “A marginal structural model analysis for loneliness: implications for intervention trials and clinical practice”. In: Journal of Consulting and Clinical Psychology 79(2), p. 225-235. 